Perinatology pISSN 2508-4887 • elSSN 2508-4895
Case report

Perinatology Vol. 31, No. 3, September, 2020 https://doi.org/10.14734/PN.2020.31.3.156

\title{
Severe Late-onset Anemia Associated with Rhesus D Alloimmunization
}

\section{Jaesung Jeon, $\mathrm{MD}$ \\ Gina Lim, MD, \\ Ki Won Oh, MD}

Departments of Pediatrics, Ulsan University Hospital, University of Ulsan College of Medicine, Ulsan, Korea
Rhesus $\mathrm{D}(\mathrm{RhD})$ alloimmunization is the most severe cause of hemolytic disease of the fetus and newborn (HDFN). However, owing to medical advances, pediatricians are likely to encounter only a few relatively mild cases. Therefore, although late-onset anemia is a well-recognized complication of HDFN, it may be overlooked in evaluations for post-discharge surveillance. Herein, we report a case of severe late-onset anemia associated with RhD HDFN. A 37-day-old male infant presented with a marked pale appearance. During the fetal period, he had received intrauterine transfusion six times for the treatment of severe anemia induced by RhD HDFN. However, during the neonatal period, he had no significant clinical manifestations, except mild jaundice. The laboratory findings on the day of presentation showed severe hemolytic anemia with reticulocytosis, which was treated with red blood cell transfusion. Our case report emphasizes that regardless of the initial disease severity during the neonatal period, regular follow-up visits and close monitoring for late-onset anemia in the first several weeks after birth are vital in infants with HDFN.

Key Words: Rh isoimmunization, Hemolytic disease of the fetus and newborn, Late-onset anemia, Intrauterine blood transfusion

\section{Introduction}

Received: 5 April 2020

Revised: 4 June 2020

Accepted: 30 June 2020

Correspondence to

KiWon Oh, MD

Department of Pediatrics, Ulsan

University Hospital, University of

Ulsan College of Medicine, 877

Bangeojinsunhwando-ro, Dong-gu,

Ulsan 44033, Korea

Tel: $+82-52-250-8861$

Fax: +82-52-250-8071

E-mail: pentawish@hanmail.net

Copyright@ 2020 by The Korean Society of Perinatology

This is an Open Access article distributed under the terms of the Creative Commons Attribution Non-Commercial License (http://creativecommons.org/ license/by-nc/4.0/), which permits unrestricted non-commercial use, distribution, and reproduction in any medium, provided that the original work is properly cited.
Rhesus $\mathrm{D}(\mathrm{RhD}$ ) alloimmunization occurs when a $\mathrm{RhD}$-negative mother produces immunoglobulin $\mathrm{G}(\mathrm{IgG})$ antibodies against the $\mathrm{RhD}$ antigen as a result of exposure to foreign $\mathrm{RhD}$ positive red cells. ${ }^{1,2}$ It remains to be the most severe cause of hemolytic disease of the fetus and newborn (HDFN) and can result in a wide range of complications, including fetal anemia and neonatal hyperbilirubinemia. ${ }^{1,2}$ However, in Korea, RhD HDFN is rarely encountered in clinical settings because of the small population of individuals with the $\mathrm{RhD}$-negative blood group and the common use of anti-D immunoprophylaxis. Moreover, with intrauterine transfusion (IUT), severe fetal anemia that leads to fetal hydrops or stillbirth can now be prevented. ${ }^{3}$ Consequently, pediatricians are likely to encounter only a few cases of relatively mild RhD HDFN. In addition, they usually focus on the prevention and treatment of early complications such as severe hyperbilirubinemia. ${ }^{4}$ Therefore, although late-onset anemia is a well-recognized complication of HDFN, it could occasionally be overlooked in post-discharge surveillance. ${ }^{1,2}$ Herein, we report the case of an infant with $\mathrm{RhD}$ HDFN who developed severe lateonset anemia on day 37 of life after having mild jaundice during the newborn period.

\section{Case}

A 37-day-old male infant with a marked pale appearance presented to the pediatric outpatient clinic. During the fetal period, he had received intravascular IUTs six times for the treatment of fetal hydrops caused by severe anemia with hemoglobin $(\mathrm{Hb})$ level of $2.9 \mathrm{~g} / \mathrm{dL}$. 
The blood groups of the patient, his mother, and his father were $A B$ $\mathrm{Rh}$-positive, B Rh-negative, and A Rh-positive, respectively. His mother was found to be Rh-negative when she conceived him. Thus, RhoGAM injections were given at around the 28th week of pregnancy and after birth. She had conceived once before, but the pregnancy ended in abortion. She had not received any anti-D immunoprophylaxis during that pregnancy. The patient was delivered through a cesarean section and weighed $3.2 \mathrm{~kg}$ at birth. His Apgar scores were 8 at 1 -min and 9 at 5 -min. At birth, complete blood cell count (CBC) showed white blood cell count

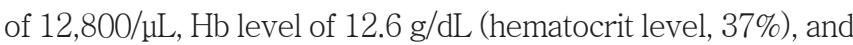
platelet count of $331,000 / \mu \mathrm{L}$. His blood group was O Rh negative as suspected in the transfusion of red blood cells (RBCs) during the fetal period. Approximately 10 hours after birth, he developed mild jaundice with total serum bilirubin (TSB) level of $9.2 \mathrm{mg} / \mathrm{dL}$. He received phototherapy for four days and was discharged on day 8, when his TSB level was $3.7 \mathrm{mg} / \mathrm{dL}$.

He was initially examined at our hospital on the 16th day of life for evaluation of anemia and jaundice. Laboratory findings showed $\mathrm{Hb}$ level of $8.3 \mathrm{~g} / \mathrm{dL}$, reticulocyte count of $0.1 \%$, and TSB level of $2.3 \mathrm{mg} / \mathrm{dL}$. As the anemia was not severe enough to require $\mathrm{RBC}$ transfusion and no findings were consistent with hemolysis, a follow-up was recommended after three months for routine checkup. However, he returned on the 37 th day of life with marked pallor. Except for the pale appearance, no specific abnormal findings such as hepatomegaly or splenomegaly were obtained on physical examination. His laboratory test results showed Hb level of $4.9 \mathrm{~g} / \mathrm{dL}$, reticulocyte count of $23.3 \%$, TSB level of $6.8 \mathrm{mg} / \mathrm{dL}$, iron level of $89 / \mu \mathrm{L}$, total iron-binding capa-

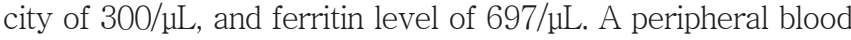

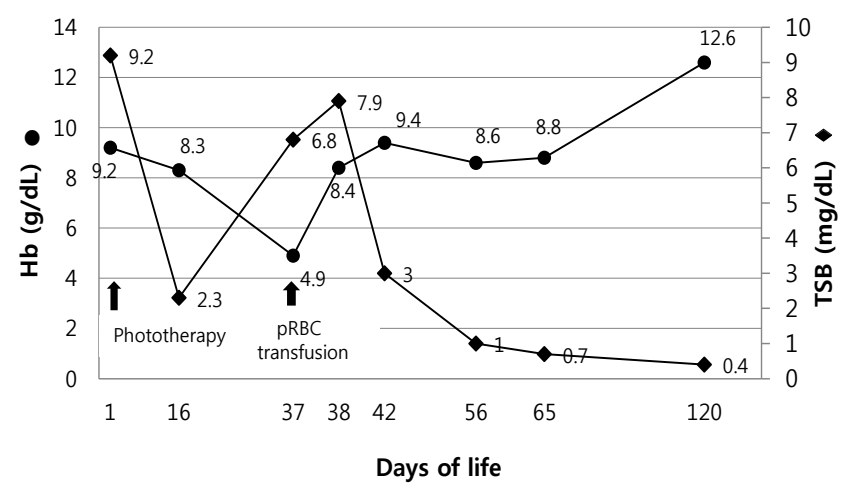

Fig. 1. Clinical summary. Changes in the patient's Hb and TSB levels. $\mathrm{Hb}$, hemoglobin; TSB, total serum bilirubin; $\mathrm{RBC}$, red blood cell. smear showed normocytic normochromic RBCs, with an increase in nucleated RBCs and marked anisocytosis. Direct and indirect Coombs tests yielded positive results, and anti-D antibodies were detected. He received transfusion with $\mathrm{O}$ Rhnegative packed RBCs. Consequently, his Hb level increased to $9.6 \mathrm{~g} / \mathrm{dL}$ by hospital day 3. During hospitalization, his TSB level was stable at $6.7 \mathrm{mg} / \mathrm{dL}$, and his reticulocyte count decreased to 13.8\%. After hospital discharge, his Hb levels at three consecutive follow-ups with 1- to 2-week intervals were 9.4, 8.6, and $8.8 \mathrm{mg} / \mathrm{dL}$, respectively. At the follow-up at four months of age, laboratory tests revealed $\mathrm{Hb}$ level of $12.6 \mathrm{~g} / \mathrm{dL}$, reticulocyte count of $0.6 \%$, and TSB level of $0.4 \mathrm{mg} / \mathrm{dL}$, and no anti-D antibodies were detected (Fig. 1).

\section{Discussion}

HDFN is a disease caused by the destruction of RBCs of the fetus or newborn by maternal erythrocyte IgG alloantibodies that have passed through the placenta. Anemia and jaundice are the main clinical findings of HDFN. ${ }^{1}$ If left untreated, progressing fetal anemia may result in hepatosplenomegaly, cardiomegaly, cardiac decompensation, and eventually, fetal hydrops and perinatal death. If the fetus survives, persistent hemolysis may lead to severe neonatal hyperbilirubinemia, which occasionally results in an irreversible brain injury known as kernicterus. ${ }^{1}$ Most cases of $\mathrm{HDFN}$ are caused by $\mathrm{ABO}$ or $\mathrm{RhD}$ incompatibility, and serious disease is mainly caused by $\mathrm{RhD}$ incompatibility. ${ }^{1}$

To prevent RhD HDFN, the anti-D immunoprophylaxis was introduced in 1968. ${ }^{1}$ The standard procedure involves injecting Rho (D) immunoglobulin (RhoGAM) once every 28 weeks of pregnancy and within 72 hours after delivery. ${ }^{1}$ After the introduction of immunoprophylaxis, the incidence of RhD HDFN decreased from $13.2 \%$ to $0.14 \%$, and the mortality rate also decreased sharply from $25 \%$ to $2 \% .^{5}$ However, the mother of our patient was not aware of her blood group type during the previous pregnancy, which ended in abortion. Therefore, she could not be treated with RhoGAM during the first pregnancy and after the abortion. The importance of providing awareness among childbearing women about their blood group type and educating women with the Rh-negative blood group about $\mathrm{RhD}$ immunoprophylaxis cannot be overemphasized. 
During the antenatal care, in addition to immunoprophylaxis, monitoring and management of fetal anemia in high-risk patients with RhD HDFN is important. ${ }^{1,2}$ The advances in Doppler ultrasonography enabled a noninvasive detection of fetal anemia by measuring the peak systolic velocity of the middle cerebral artery (MCA-PSV). For the management of severe fetal anemia, intravascular IUT has been the cornerstone since it was first described in $1981 .^{3}$ IUT should be considered when clinical assessment indicates fetal anemia. IUT is recommended when the MCA-PSV Doppler measurement exceeds 1.5 multiples of the median for gestational age or when signs of fetal edema appears. ${ }^{3}$ Most studies have shown perinatal survival rates of $>90 \%$ with IUTs treatment. Moreover, in the long-term followup, $>95 \%$ of the infants treated with IUTs showed normal neurodevelopmental outcomes. In our case, the patient's severe fetal anemia with $\mathrm{Hb}$ level of $2.9 \mathrm{~g} / \mathrm{dL}$ was successfully managed using six courses of IUT.

During the neonatal period, the main issue with RhD HDFN is the management of hyperbilirubinemia., ${ }^{1,2}$ As severe hyperbilirubinemia in the immediate postnatal period can cause bilirubininduced neurologic dysfunction, it should be treated with intensive phototherapy and exchange transfusions when the bilirubin concentration reach critical levels. ${ }^{1,2}$ In our case, unlike its severity in the fetal period, hyperbilirubinemia was mild in the early neonatal period and could be treated with phototherapy alone. This could be explained by the fact that at birth, his circulation was comprised primarily of $\mathrm{O}$ Rh-negative $\mathrm{RBC}$ s originating from IUTs and severe hemolysis did not develop, even with the presence of alloantibodies.

Late-onset anemia, which develops after seven days of age, is a well-known complication of RhD HDFN. It is found in 83\% of neonates with $\mathrm{RhD} \mathrm{HDFN}$, and the incidence is highest among infants who received IUTs. It commonly develops between two and six weeks after birth and usually resolves by the third month of life. ${ }^{1}$ It is divided into late hyporegenerative anemia and late hemolytic anemia. ${ }^{1}$ Late hyporegenerative anemia is characterized by ineffective erythropoiesis with low reticulocyte counts. Although intramedullary destruction of erythrocyte precursors, bone marrow suppression from IUTs, and erythropoietin (EPO) deficiency have been proposed as the main causes of late hyporegenerative anemia, the pathogenesis is not entirely understood. In fact, IUTs seem to increase the risk of late hyporegenerative anemia, which was described before IUTs became available. Moreover, EPO levels are not always low in patients with late hyporegenerative anemia. In contrast to late hyporegenerative anemia, late hemolytic anemia is characterized by active bone marrow erythropoiesis compensating for the shortened erythrocyte survival, with age-appropriate or elevated reticulocyte counts. Late hemolytic anemia is thought to be caused by a combination of antibody-mediated hemolysis, natural decrease in Hb level toward the physiologic nadir, and rapid expansion of the intravascular volume in a growing infant. It is commonly associated with elevated bilirubin levels. ${ }^{6}$ In our case, the $\mathrm{CBC}$ test performed two weeks after birth showed a mild decrease in $\mathrm{Hb}$ level and a severe decrease in the reticulocyte count. This may have resulted from the shortened half-life of transfused RBCs during the fetal period and the bone marrow suppression caused by IUTs. At that time, a short-term follow-up had to be considered to observe the changes in the $\mathrm{Hb}$ level and reticulocyte counts; however, this seems to have been overlooked. At three weeks after the pediatric outpatient clinic visit, because of the patient's pale appearance, $\mathrm{CBC}$ tests were performed and revealed severe anemia with an increase in reticulocyte counts, reflecting the ongoing $\mathrm{RBC}$ hemolysis. This might have been caused by the residual maternal alloantibodies in his circulation, which resulted in prolonged ongoing anemia. ${ }^{6,7}$ As maternal alloantibodies can be active for 6 to 10 weeks in the neonate and recovery of erythropoiesis may be delayed as an effect of IUTs, weekly monitoring of Hb level and reticulocyte count should be checked in all infants with RhD HDFN until 3 months of age. ${ }^{8}$

The primary treatment for late-onset anemia in $\mathrm{HDFN}$ is $\mathrm{RBC}$ transfusion. In cases of late hyporegenerative anemia, recombinant $\mathrm{EPO}$ has been the most commonly used treatment to prevent late-onset anemia and reduce the need for RBC transfusion. However, the effectiveness of EPO is still controversial, and the optimal dose and time of therapy initiation have not been defined. ${ }^{5}$ In addition EPO and other pharmacotherapeutic agents such as folate, iron, and vitamin E may have beneficial effects for the prevention or treatment of late-onset anemia in HDFN. However, the available data are insufficient to support the supplementation of these agents in HDFN. Especially infants with HDFN already have high iron storage due to the combination of prolonged hemolysis and treatment with multiple IUTs and 
postnatal RBC transfusion; thus, iron supplementation should be used cautiously in these infants. ${ }^{1}$ In our case, late hemolytic anemia was the main cause, and fortunately, after the RBC transfusion, the patient's Hb level was stabilized.

Our case highlights that severe late-onset anemia could be a complication of HDNF, regardless of the disease severity in the early neonatal period. Therefore, regular follow-up visits and close monitoring for late-onset anemia once or twice a week are important in the first several weeks after birth.

\section{References}

1) Ree IMC, Smits-Wintjens VEHJ, van der Bom JG, van Klink JMM, Oepkes D, Lopriore E. Neonatal management and outcome in alloimmune hemolytic disease. Expert Rev Hematol 2017;10:607-16.
2) De Haas M, Thurik FF, Koelewijn JM, van der Schoot CE. Haemolytic disease of the fetus and newborn. Vox Sang 2015;109:99-113.

3) Zwiers C, van Kamp I, Oepkes D, Lopriore E. Intrauterine transfusion and non-invasive treatment options for hemolytic disease of the fetus and newborn - review on current management and outcome. Expert Rev Hematol 2017;10:337-44.

4) Jadala HV, Pooja V, Raghavendra K, Prithvish CM, Srinivas B. Late onset severe anemia due to rhesus isoimmunization. Int J Contemp Pediatrics 2016:3:1472-73.

5) Zuppa AA, Alighieri G, Calabrese V, Visintini F, Cota F, Carducci C, et al. Recombinant human erythropoietin in the prevention of late anemia in intrauterine transfused neonates with Rh-isoimmunization. J Pediatr Hematol Oncol 2010;32:e95-101.

6) al-Alaiyan $S$, al Omran A. Late hyporegenerative anemia in neonates with rhesus hemolytic disease. J Perinat Med 1999;27:112-5.

7) Strand C, Polesky HF. Delayed anemia in erythroblastosis fetalis. Minn Med 1972;55:439-41.

8) Mitchell S, James A. Severe late anemia of hemolytic disease of the new born. Paediatr Child Health 1999;4:201-3. 